\title{
Museum augmented interface for historical scale models: towards a new way for cultural heritage promotion
}

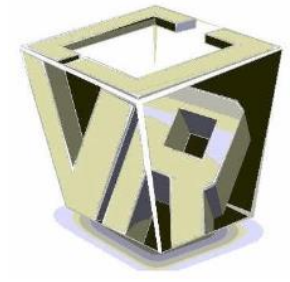

\author{
Benjamin Hervy ${ }^{1}$, Florent Laroche ${ }^{1}$, Jean-Louis Kerouanton ${ }^{2}$, Alain Bernard ${ }^{1}$, \\ Christophe Courtin ${ }^{3}$, Bertrand Guillet ${ }^{3}$, Laurence D'haene ${ }^{3}$, Arnaud Waels ${ }^{4}$ \\ 1 LUNAM Université, Ecole Centrale Nantes, IRCCyN UMR CNRS 6597 \\ 2 LUNAM Université, Université de Nantes, CFV EA1161 \\ 3 Château des ducs de Bretagne, Musée d'histoire de Nantes \\ 4 « Dev-o-cité » association
}

\begin{abstract}
In this paper, we describe an interactive museum application dedicated to historical scale models. This comes from a joint work between multidisciplinary teams: industrial engineering researchers, historians, museum curators and interactive interface designers. We present here the result of the project, based on scientific methodology. Results include system architecture, hardware and software, some use cases and user evaluation figures. This paper also underlines some methodology issues that illustrate future possibilities.

Categories and Subject Descriptors - D.2.2 [Software engineering]: Design Tools and Techniques - user interfaces.

H.5.2 [Software engineering]: User interfaces - Usercentered design.
\end{abstract}

\section{J.5 [Computer applications]: Arts and humanities.}

General Terms - Documentation, Design, Experimentation, Human Factors

Index Terms - Museology, User interface, History, Knowledge management

\section{INTRODUCTION}

The increasing availability and performance of digital technologies is making museum collection promotions more interactive. Cultural heritage benefits greatly from both acquisition techniques and promotion applications. However, accurate interactive experience of history does not only rely on innovative technologies but also on innovative uses of low-cost digital material.

One of the most crucial issues in cultural heritage promotion is the process of curation. From heterogeneous data acquisition to appropriate visitor experience, a complex methodology is involved to achieve scientific purposes and cultural promotions.
Methodological aspects of this work have already been published in detail. In [1], we explained the process of historical scale model acquisition and the combination with semantic information. We explained in [2] how knowledge modeling is important to avoid one-shot application and we introduced the advanced visualization possibilities to explore cultural heritage history thanks to virtual reality.

In this paper, we focus on an innovative use of digital technologies to promote cultural heritage. The museum context is related to a 100-year old scale model designed in 1899 to promote Nantes (French city) harbor activity.

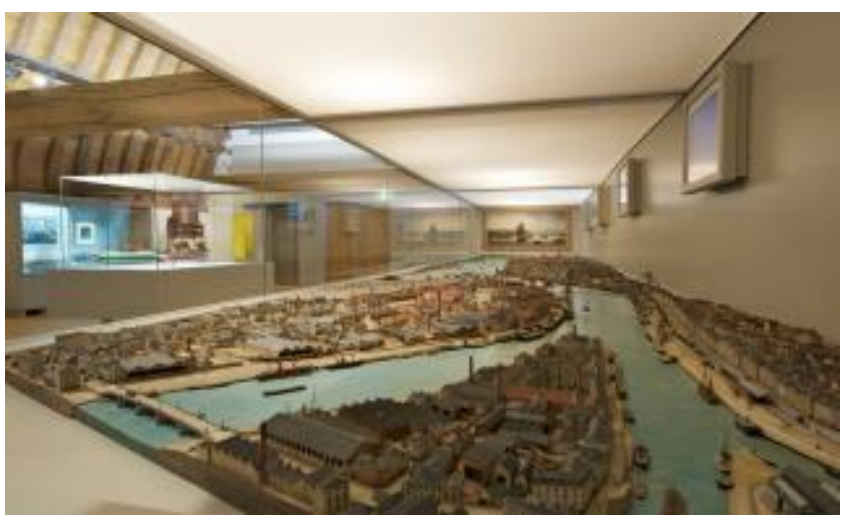

Fig. 1: Nantes harbor scale model in the History Museum

Due to its dimensions $\left(\sim 18 \mathrm{~m}^{2}\right)$, this scale model is a precious witness to historians. Its scale is 1:500. It is currently exposed in the permanent exhibition of Nantes History Museum in the "Château des ducs de Bretagne" and only little information is displayed to visitors: a 10-line label and 3 archive movies. Moreover, nobody can touch the object, for obvious conservation purposes.

We propose an augmented visualization system to allow visitors to virtually manipulate the scale model. This system has been designed in a reproducible way, so that the approach can be used to promote other scale models. Application source codes, database schemas and historical material will be available for reuse purpose. 
Cultural heritage comprehension and promotion would benefit from formal methodology. In this paper we describe a process to promote one kind of $\mathrm{CH}$ object (scale models), but the methodology could be applied to the whole museum collection. It can also enhance museum discourse as far as museum objects can be connected on a historical point of view.

\section{RELATED WORKS}

A virtual 3D mock-up is a widely used artifact for cultural heritage promotion and understanding. It can be used to explore archaeological sites, or for hypothesis analysis. Some interesting projects combine 3D visualization for? scientific historical purposes [3]. Such applications provide important tools for researchers to explore physical space in its historical context. Virtual reality technologies such as the "3D panoscope 3D" [4] can also be used to provide an immersive experience to the visitor. Interactivity such as immersive experiences is considered useful when dealing with cultural heritage comprehension [5] [6] [7].

As far as scale models are concerned, many projects already exist [8] [9] [10]. But most of them concern blank models on which it is possible to make video mapping and other visual effects. Very few deal with historical "plan-reliefs". Some have emerged worldwide. The following table summarizes the different project characteristics.

\begin{tabular}{|l|l|l|l|l|l|l|}
\hline & Nantes & Liège & Toul & Prague & Beijing & Rome \\
\hline $\begin{array}{l}\text { Performance } \\
\text { date }\end{array}$ & 1898 & 1900 & 1846 & $\mathbf{1 8 2 6}$ & 1950 & 1970 \\
\hline $\begin{array}{l}\text { State of } \\
\text { conservation }\end{array}$ & Good & Good & Bad & Good & Good & Good \\
\hline Scale & $1: 500$ & $\mathbf{1 : 1 2 0 0}$ & $1: 600$ & $1: 480$ & $1: 1000$ & $1: 250$ \\
\hline Surface(m²) & 17 & 1 & 39 & 20 & 75 & 278.4 \\
\hline Acquisition & Laser & Laser & $\begin{array}{l}\text { Photographs } \\
\text { \& Laser }\end{array}$ & Stereoscopy & $\begin{array}{l}\text { Structured } \\
\text { light \& } \\
\text { Stereoscopy }\end{array}$ & Laser \\
\hline
\end{tabular}

Table 1: Existing historical scale model digitization projects

These figures illustrate a real need for a museum when dealing with such objects. Besides the design of interactive application for visitors, there are great challenges to deal with.

Here, we focus on the design process of the museum visitors application based on physical objects and information acquisition. First, we need to define the scope of the discourse in space and time. The space delimitation is quite easy as it is delimitated by the object itself. The time period can be more complicated as we can broaden the historical study. We chose to limit the research area to the epoch of this object: from the end of the $19^{\text {th }}$ century to the beginning of the $20^{\text {th }}$ century. But we provide to the system the ability to handle any kind of element in time. From a historical point a view, this is a great advantage as it allows future research to be included and to be compared with existing material.

Considering the promotion challenge for visitors, we did not find any existing satisfying application for such objects. Historical scale models are almost never integrated in an interactive application for museums. Those who actually designed visualization facilities focus on one-shot applications without the possibility of content evolution.

Our approach is mainly based on a semantic historical content. We then connect any available source (graphical, textual, audio, video), geographical information and virtual 3D data. 3D data comes from the scanning of the object [12]. Finally, we design an augmented system based on a multitouch interface and video projection, both connected to specific software and to a dedicated open database.

\section{PHYSICAL SYSTEM ARCHITECTURE}

The proposed "augmented scale model" system is based on 5 main components:

- The physical scale model

- The virtual 3D model

- One or several multitouch screens (depending on the size of the object). We choose 30 inch screens for accessibility issues and ergonomic purposes.

- $\quad$ One or several video projectors (depending on the size of the object)

- Web accessible database

- User graphic interface

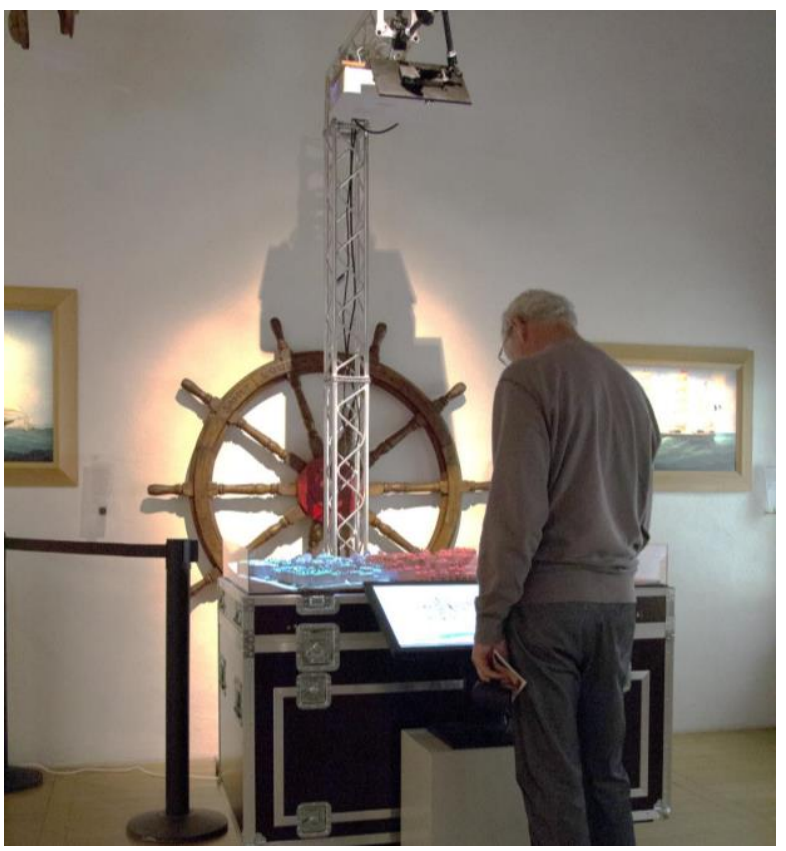

Fig. 2: Proof-of-concept proposal for an "augmented scale model"

Human machine interface relies on the connection between these 6 elements. Visitors can virtually explore the city through the multitouch screen. The main interface is composed of two highdefinition pictures (8000px width): human-like view (using image stacking) and orthophoto. The two pictures are taken thanks to semi-automated photo shooting.

\subsection{Database schema and implementation}

A database schema is based on four main classes:

- $\quad$ Semantic data provided by analysis from historians. It is modeled as a historical description sheet.

- Related historical sources used by historians. Sources can be bibliographic references, graphical assets or any other oral, video archive.

- Semantic relationships between points of interest. These links provide the user with the ability to navigate through the content. 
- $\quad$ Space and time information. The Nantes harbor scale model is limited in space and time. The represented city is contemporary to the beginning of the twentieth century.

\subsection{Interactive software description}

The multitouch designed application is written in Python, with the free software Kivy ${ }^{1}$ framework. Kivy is widely used to design multitouch applications and is portable on every platform.

It allows gestures with several fingers: selecting, scaling, panning, and rotating.

Our software connects to the database and retrieves metadata and values in JSON format. It can display a window with a 3D virtual model thanks to the Panda3D open-source library ${ }^{2}$.

Our application uses a virtual 3D model to automatically detect scale model parts that need to be illuminated using the Polygon python library ${ }^{3}$. Moreover, it also uses the virtual 3D model to cut a newly inserted area into the main interface picture.

For research purposes, we designed a proof of concept (Fig. 2) of the proposed system. It is based on a smaller scale model, from the same author and the same epoch, that also belongs to the Nantes History Museum.

\section{DESIGN PROCESS OVERVIEW}

An analogy with an iceberg easily gives an overview of the underlying process. Our approach is mainly user-centered. But our global system users are not only museum visitors. They are also museum professionals and other experts such as historians, and archaeologists.

Therefore, we do insist on a knowledge organization system. This is crucial to design a proper final application [12].We did not want to design a one-shot device but rather imagine a global information system for the museum. This is important to understand our process described in Fig.3.

\subsection{Project resources}

In such a project the resources involved, and especially human resources are significant. Here is a non-exhaustive taxonomy of involved skills:

- Curators who define the scope of the project. They contribute to the related knowledge of the object and work on museum discourse.

- Museum mediators who work on museum discourse for the visitors.

- Historians who work with curators on the scientific content. They lead archive research and

- IT engineers who work on an information system design and 3D acquisition.

- Software developers who work on concrete implementation

- $\quad$ Graphic designers

- Museum technical teams to maintain the application.

A trans disciplinary research team is mandatory to build a museum discourse on top of a robust scientific corpus. It allows

\footnotetext{
${ }^{1}$ http://kivy.org/

${ }^{2}$ https://www.panda3d.org/

${ }^{3}$ https://pypi.python.org/pypi/Polygon/2.0.4
}

the system to be scalable: other end-user applications can be designed on top of this digital heritage reference model.

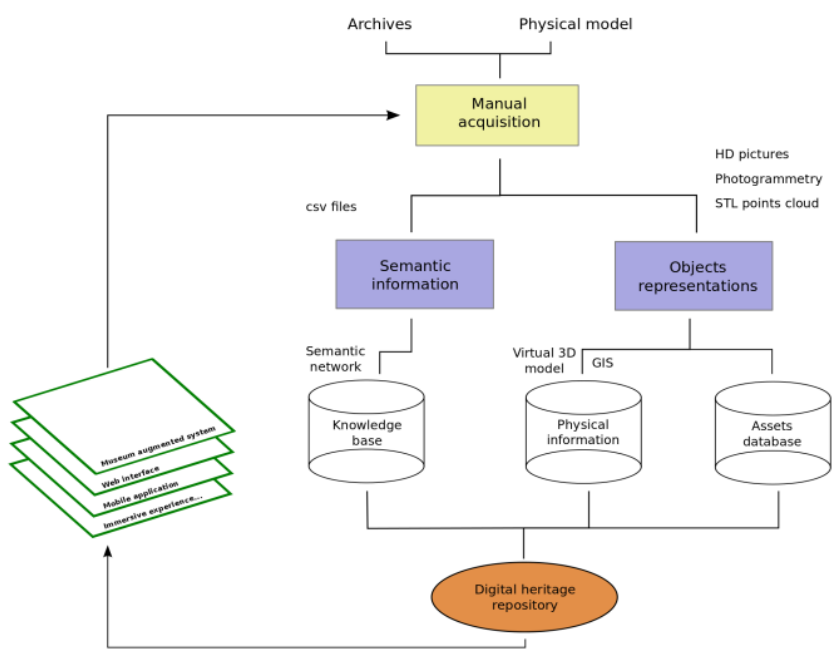

Fig. 3: Pipeline. We separate semantic information consisting of contextual knowledge and geographical data (or any graphical representation). End-user applications such as the proposed augmented scale model framework are connected to the virtual record. We can finally capitalize new knowledge coming out from the use of existing applications.

\subsection{Data acquisition}

\subsubsection{Physical object acquisition: 3D scanning}

The global process consists of merging semantic and physical information into a virtual artifact. In the case of urban city models, it is called a 3D virtual city model (3DVCM) [1].

A scale model 3D digitization (Creaform HandyScan 3D) provides an STL file composed of almost 100 million points. Many topological issues still have to be treated at this step: holes, file mergers, missing parts and so on. Post-treatment (using Geomagic software) to solve these issues is described in [11].

Once the virtual 3D model is properly treated, it can be used for several purposes:

- Virtual world generation could lead to virtual immersive experience in old Nantes city harbor. This implies a texturing step that is not covered in the scope of this paper.

- Semi-automatic geo-location is interesting for the retrieval of geographical information. This could lead to geo-located mobile applications for example. In our case, this is not fully implemented because of the poor quality of the extracted 2D map. The other main issue is the incorrectness of the scale model regarding the actual city.

- Connection between touchscreens, software applications and video-projections on the physical object is the main use case for instance. When an area is selected on the main user interface, the corresponding virtual area is detected in the virtual model. Then, the system retrieves the corresponding area of interest in the database to highlight it on the scale model in front of the visitor. 


\subsubsection{Physical object acquisition: photogrammetry} In addition to the $3 \mathrm{D}$ digitization, photo acquisition is done to reproduce the view a visitor would have of the object in front of him. This step is semi-automatic and requires a home-made traveling system to move the camera. Two pictures are taken for the application purpose: a $45^{\circ}$ oriented picture using an image stacking technique and an orthophoto using photogrammetry.

For both view points, the camera is programed to cover every part of the scale model. 25 photos are taken for each camera position with a different focal length each time. 20000 photos are necessary to provide the 8000 pixel width final picture. The final picture resolution guarantees that the visitor will be able to zoom into the picture.

It also allows us to make the link between geographical parts and semantic data in the database. Every part of the city (building, place, street) on the physical object is stored as a polygon in the scale model coordinate system.

Polygons can be drawn manually on our dedicated web interface, on top of the picture, or can be retrieved semiautomatically for the 2D map extracted from the 3D model (this has not yet been fully implemented). Polygons coordinates are stored in WKT (well-known text) to PostGIS software.

A mapping of object IDs allows the system to identify what part of the museum scale model is linked to which historical object database.

For the future, we are working on the possibility to semiautomatically derive geo-located data from these local coordinate system polygons.

\subsubsection{Semantic data acquisition}

Historians have done research in archives for about 500 points of interest. They can be related to a company, a building (shipyard), a person (an employee) or even a theme ("Shipment industry at the beginning of the twentieth century").

Each one of these subjects is documented thanks to historical sources that can be either texts and/or graphics. In addition, they are also linked together thanks to metadata and especially keywords.

For every subject, we have a dedicated sheet containing metadata (title, description, address, keywords), paradata (historian information, annotation) for scientific methodology, associated sources and spatio-temporal information. For example, we can specify that the "Chantiers Dubigeon" company was at place "A" from date 1 to date 2 . We can also mention the precision for time information. Space precision is not handled for instance.

\subsection{Data modeling}

Our conceptual model is based on the DHRM (Digital Heritage Reference Model), a meta-model for industrial heritage analysis [4] [12].

In our context, we deal with semantic information (items of interest and relationships between them), geographic information with time specification (location of physical parts on the scale model and possibly geo-location information), and other assets such as digitized paintings, postcards, photos, etc.

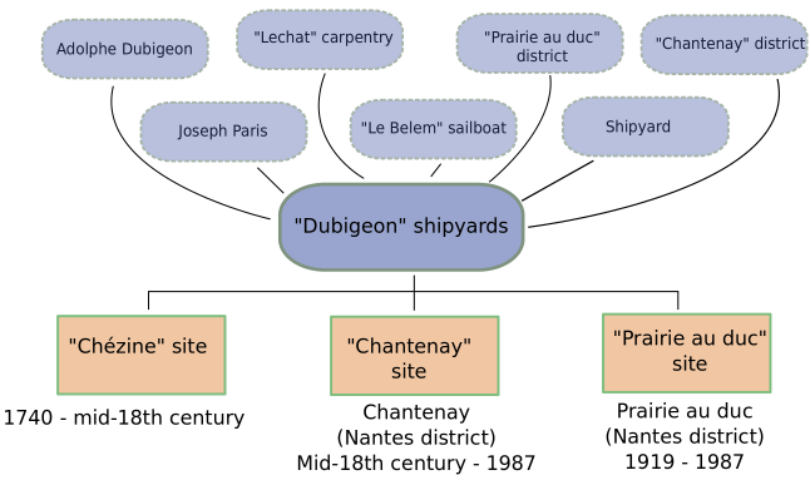

Fig. 4: "Dubigeon" shipyards instantiation example

All these concepts have been modeled in a relational database. For each class, we store dedicated metadata. Graphical assets metadata come from the existing museum collection databases. Semantic information metadata (title, address, description) are adapted from the museum collection database. For design purposes, we add metadata (summary, formatted keywords, coordinates in the physical object coordinate system) and relations based on keywords.

The object class attributes basic metadata such as title, description, address, and keywords. This class could be extended with reference models such as CIDOC-CRM to specify the typology of objects involved.

The relationship class stores the links between objects based on keywords. Some metadata can be added to specify the relationship properties: annotation, the beginning and end dates of the relationship. Once again, the model could be extended to take metadata from external schemas (Dublin Core, CIDOCCRM) into account.

Geographic information is time dependent. This allows the system to possibly be scalable: current data is approximately situated between 1880 and 1930, but we could imagine extending the time span. An object can have several geographical positions over time.

We implemented the model using free software Postgres. Geographic information is stored in PostGIS extension. For the semantic data, tests have also been done using Neo4j graph database with complete success. But this is still at an experimental stage and could be used for future research purposes (identification of historical paths of interest).

\subsection{Web interface}

A web interface, written in standard web languages (HTML, CSS PHP, Javascript) with CodeIgniter framework, allows researchers to upload their data, and museum teams to edit and access the data.

A moderation and validation process has been decided to guarantee the quality of content. Once the data is validated, it is available online to everyone. Registered users can also propose new content such as postcards, photos or give information on new elements depending on their access level. For example, a basic user can only upload assets whereas a researcher can add new information about a building. 


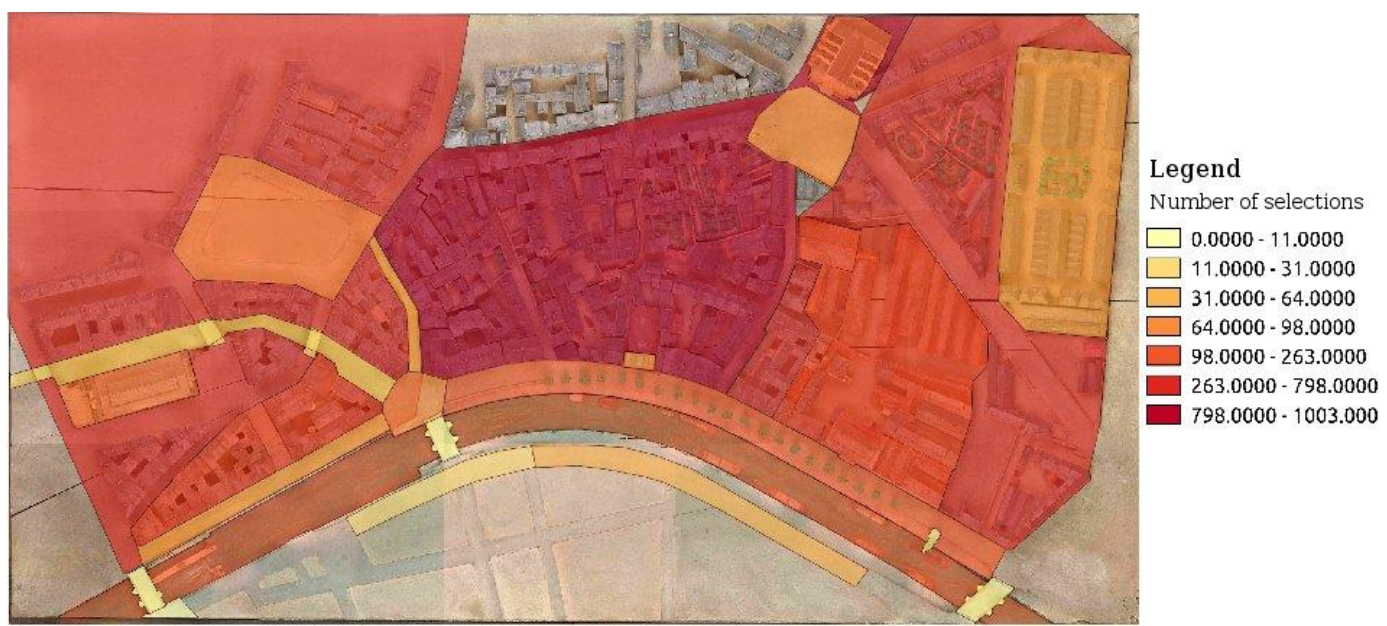

Fig. 5: Visitor selections during test exhibition

\section{USE CASES}

\subsection{Visitor use case}

The basic use case concerns a single visitor. He can navigate through the main interface by zooming, panning, and selecting a place.

The main behavior is based on two steps. The scale model has been divided into three main neighborhoods. Thus, any visitor action first redirects him to one of these neighborhoods. Then, the sub-parts of the neighborhood are highlighted and can be selected.

Once he selects an area, the system displays related content available in a carousel. Graphical resources can be zoomed and texts can be resized depending on user abilities. Some related points of interest are also underlined to give some navigation clues for the visitor. Non-geographical elements are displayed at the bottom of the screen. They allow visitors who select a particular bridge or street to see all the bridges/streets on the physical scale model. This leads to serendipitous navigation based on our semantic network.

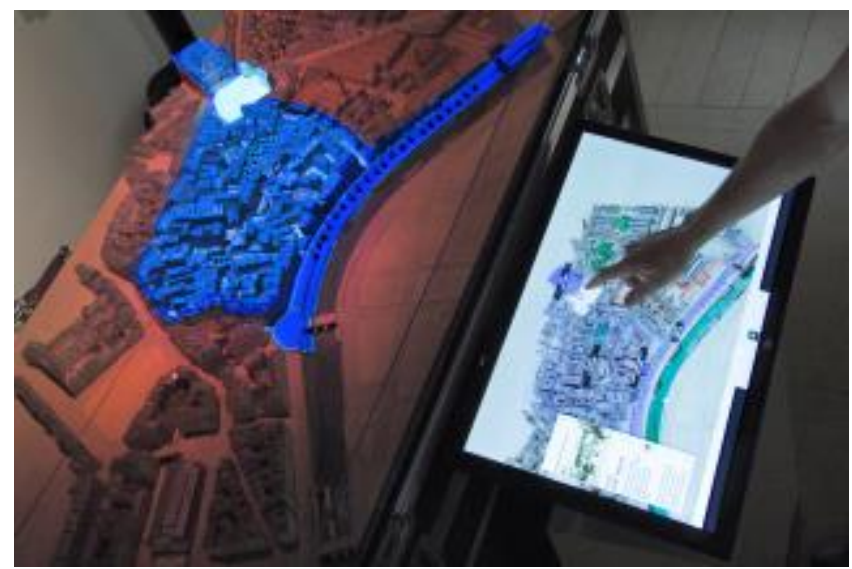

Fig. 6: Area selection and lighting feedback

We do not force any scenario; everyone can do research on any available element.

\subsection{Museum guides use case}

A specific back-office has been built next to the museum system to help the museum team in their guided tours. Indeed, museum guides can choose the content to display in advance.

Doing so, they will take control of the desired screens (possibly all of them) in front of the object thanks to an RFID badge. An RFID chip contains every ID previously selected by the museum personnel. The system will then be able to display selected items on the screen to the group of visitors. It will help the guide to illustrate his/her discourse.

\section{EVALUATION \& OUTCOME}

For research purposes, we have designed a prototype of the proposed system. It is based on a smaller scale model, from the same author and the same epoch, that also belong to the Nantes History Museum.

Thanks to smaller dimensions, we only deal with 50 points of interest. The acquisition and data modeling techniques are the same as described above.

For project management purposes (iterative improvements), we set up three evaluation stages:

- One with 20 museum professionals who were first asked about their digital tool experience and expectations.

- One by observing 80 museum visitors in a real situation inside the museum during an afternoon. They were free to use the system, and we asked them to react after their experience.

- $\quad$ One during a 4-day exhibition. The exhibition was about digital heritage. A simple script written in Python recorded visitor actions and time spent. No distinction of visitor age or gender was made.

These three different evaluation studies give us some significant clues on both the ergonomic aspect and the cultural mediation impact of such a system.

\subsection{Evaluation results}

We describe here what appear as significant elements when dealing with cultural heritage promotions. In the next two 
sections, we focus on the social consideration based on our observations, and on functional aspects.

\subsubsection{Qualitative observations}

The results of our observations are interesting. A proposed system makes people understand objects and city history thanks to the pooling of information and assets. Moreover, it creates group emulation. We observe that people use different methods for navigation: quick surveys of available content, methodic access, full text reading or only graphical assets. Video projection feedback on the object catches the visitor's eye.

\subsubsection{Quantitative observations}

Thanks to our monitoring system, we could retrieve some quantitative data from four full days of use. Fig. 5 illustrates the percentage of actions for each part of the scale model.

We can clearly identify the three main neighborhoods. The central one is preferred, probably due to its easier access. This kind of geographical access gives benefit to large areas. That is why our system is composed of 2 steps: every single place of interest will be highlighted, even the smallest one. No element is left aside.

In addition, it is interesting to underline the percentage of selected assets when a selection occurs. In such a case, a carousel displays all available historical material (3D view, description text, digitized content). Despite the prior position of a description text in the carousel, almost $70 \%$ of selected items are images. And during the 4-day exhibition, $50 \%$ of the total number of images were seen.

Finally, as far as time consumption is concerned, the 4-day exhibition shows that people approximately spend only a few seconds $(\sim 5)$ on displayed material. The observation day in the museum told us that visitors only spent 2 to 5 minutes on the system.

\subsection{Perspective}

Based on our results, we could define some future improvements and goals. On the one hand, more use case scenarios can be defined for disabled people, scholars, foreigners, etc. On the other hand, we can imagine interfaces to answer other cultural mediation means: augmented reality tours in the real city, immersive tours at home, and dedicated research interface for experts.

\section{CONCLUSION}

We propose an approach for improving historical scale model promotion. The method, applied on 2 similar scale models has proven the importance of multi-disciplinary work and a formal process based on a digital heritage reference model.

This approach shows the scalability of the method that could lead to a global museum collection management system. This system would connect museum objects together, provide information to many multi-modal end-user applications, and allow access to different museum professionals and experts.

\section{REFERENCES}

[1] Hervy, B., Billen, R., Laroche, F., Carré, C., Servières, M., Van Ruymbeke, M., Kerouanton, J.-L. (2012). A generalized approach for historical mock-up acquisition and data modelling: Towards historically enriched 3D city models. In T. Leduc, G. Moreau, \& R Billen, Usage, Usability, and Utility of 3D City Models - European COST Action TU0801 (p. 02009). France: EDP Sciences.
[2] Hervy, B., Laroche, F., \& Bernard, A. (2012). An information system for driving the future plm for museum: the DHRM, Digital Heritage Reference Model. In ASME 2012 11th Biennial Conference On Engineering Systems Design And Analysis - ESDA 2012. Nantes, France.

[3] Michel, A. P. (2009). La reconstitution virtuelle d'un atelier de Renault-Billancour: sources, méthodologie et perspectives. Documents pour l'histoire des techniques, (18), 23-36

[4] Philippe Dubé, \& Courchesne, L. (2012). Un lieu qui parle: la modélisation architecturale 3D vers une quatrième dimension. Hereditas Monasterium, 1, 129-137.

[5] Flynn, B. (2008). Augmented visualisation: Designing experience for an interpretative cultural heritage. In Information Visualisation, 2008. IV'08. 12th International Conference (pp. 447 - 452). IEEE.

[6] Roussou, M. (2000). Virtual Heritage: From The Research Lab To The Broad Public. In F. Niccolucci (Ed.), Virtual Archaeology: Proceedings of the VAST Euroconference (pp. 93-101).

[7] Champion, E. (2011). Augmenting the Present With the Past. In Playing with the Past (pp. 157-176). London: Springer London.

[8] Chevrier, C., Jacquot, K., \& Perrin, J.-P. (2010). 3D modelling of a town scale model. In Third International Conference EuroMed 2010 dedicated on Digital Heritage. (pp. 99-107).

[9] Gabriele Guidi; Bernard Frischer; Monica De Simone; Andrea Cioci; Alessandro Spinetti; Luca Carosso; Laura L. Micoli; Michele Russo; Tommaso Grasso; Virtualizing ancient Rome: 3D acquisition and modeling of a large plaster-of-Paris model of imperial Rome. Proc. SPIE 5665, Videometrics VIII, 119 (2005)

[10] Billen, R., Blain, P., Donneau, O., Habraken, S., Renotte, Y., \& Van Ruymbeke, M. (2009). Virtual model of the city of Liège in the eighteen century - "Virtual Leodium." In J. Verly (Ed.), Proceedings of 3D Stereo MEDIA 2009, International 3D Stereo Film and Technology Festival (3D Stereo MEDIA). Liège.

[11] Laroche, F., Lefevre, D., Servières, M., Hervy, B., Bernard, A. (2012). Intelligent Reverse-Engineering Segmentation: Automatic Semantic Recognition of Large 3D Digitalized Cloud of Points Dedicated to Heritage Objects. ASME 2012 11th Biennial Conference on Engineering Systems Design and Analysis, Jul 2012, Nantes, France.

[12] Laroche, F., Bernard, A., \& Cotte, M. (2008). Advanced Industrial Archaeology: A new reverse-engineering process for contextualizing and digitizing ancient technical objects. Journal Virtual and Physical Prototyping, 3, pp.105-122 